\title{
Conocimiento especializado sobre gráficos estadísticos de futuras maestras de educación infantil
}

\author{
Danilo Díaz-Levicoy ${ }^{1,4^{*}}$, Marjorie Samuel ${ }^{2}$ y Francisco Rodríguez-Alveal ${ }^{3}$ \\ (1) Facultad de Ciencias Básicas, Universidad Católica del Maule, Avda. San Miguel 3605, Talca-Chile. \\ (correo-e: dddiaz01@hotmail.com) \\ (2) Facultad de Educación, Universidad Autónoma de Chile, Cinco Pte. 1670, Talca-Chile. \\ (correo-e: marjorie.samuel@uautonoma.cl) \\ (3) Facultad de Educación y Humanidades, Universidad del Bío-Bío, Avda. Brasil 1180, Chillán, Chile. \\ (correo-e: frodriguez@ubiobio.cl) \\ (4) Centro de Investigación en Educación Matemática y Estadística, Universidad Católica del Maule, Avda. San Miguel \\ 3605, Talca-Chile.
}

* Autor a quien debe ser dirigida la correspondencia.

Recibido Ene. 22, 2021; Aceptado Mar. 16, 2021; Versión final May. 17, 2021, Publicado Oct. 2021

\begin{abstract}
Resumen
En este estudio se busca caracterizar el conocimiento especializado sobre gráficos estadísticos por 102 futuras maestras de educación infantil en Chile. Se sigue una metodología cualitativa, de nivel descriptivo, basada en el análisis de contenido. Particularmente, se reportan cuatro ítems que valoran aspectos de este subdominio del modelo MKT (Mathematical Knowledge for Teaching) relacionados con manifestar un conocimiento profundo y específico, y representar con exactitud ideas sobre gráficos estadísticos. Los resultados muestran que las participantes presentan errores en la definición de gráfico estadístico, identificación de conceptos vinculados al gráfico de barras, así como a los cálculos y predicciones basados en la información representada. Se concluye que las futuras maestras de educación infantil manifiestan escasos conocimientos sobre gráficos estadísticos, definidos en los estándares orientadores para estas carreras en el contexto chileno.
\end{abstract}

\section{Specialized knowledge on statistical graphs by future teachers of early childhood education}

\begin{abstract}
This study aims to assess specialized knowledge on statistical graphs by 102 early childhood education future teachers from Chile. A qualitative, descriptive level methodology is employed, based on content analysis. In particular, four items that assess aspects of this subdomain of the MKT (Mathematical Knowledge for Teaching) model are reported. The items relate to expressing deep and specific knowledge and to representing ideas accurately on statistical graphs. The results show that teachers commit errors when defining statistical graphs, when identifying concepts linked to bar graphs, and when making calculations and predictions based on the information represented on graphs. It is concluded that future teachers of early childhood education lack specialized knowledge on statistical graphs as defined by Chilean standard guidelines for early childhood education degrees.
\end{abstract}

Keywords: future teachers; early childhood education; statistical graphs; knowledge; specialized 


\section{INTRODUCCIÓN}

En la actualidad, la estadística es considerada una disciplina transversal y relevante dada sus aportaciones en diferentes áreas del conocimiento (científicas, sociales y humanísticas) (Molina-Portillo et al., 2019). En este sentido, es importante destacar su utilidad en variadas actividades de la vida cotidiana, por ejemplo, la presencia de las medidas de resumen y las representaciones gráficas y tabulares en los medios de comunicación (Gal, 2011; Jurečková y Csachová, 2020; McConway, 2016). Estos elementos son parte de la cultura estadística, es decir, del derecho que tiene todo ciudadano para leer e interpretar información de tipo estadística a la que acceden en situaciones de la vida cotidiana, cívica y profesional (del Pino y Estrella, 2012). Por otra parte, para Weiland (2017), la cultura estadística va más allá de evaluar críticamente la información a la que se tiene acceso, debe ser un lente para ver el mundo.

Gould (2017) destaca el doble rol del futuro ciudadano, quien además de consumir críticamente información estadística, debe ser capaz de producirla y comunicarla. Además, Lipič y Ovsenik (2020) relevan que contar con una cultura estadística facilita una participación activa en diferentes situaciones de la vida cotidiana, permitiendo al individuo responder eficazmente a los cambios a los que se enfrenta. En Chile, siguiendo tendencias internacionales, se ha incorporado la enseñanza de la estadística dentro de la asignatura de matemática desde los primeros cursos de Educación Primaria (Díaz-Levicoy et al., 2019). Avanzando aún más en estas ideas, Alsina (2017) establece tres motivos interrelacionados para justificar la enseñanza de la estadística desde la Educación Infantil: "1) La importancia de garantizar una educación de alta calidad que se ajuste a los cambios sociales; 2) La importancia de las matemáticas en general, y de la estadística y la probabilidad en particular, en el desarrollo integral de los niños; 3) Relevancia de la alfabetización estadística y probabilística".

Este contexto plantea desafíos importantes a las instituciones formadoras de profesores de diferentes niveles, en particular las educadoras de párvulos (o maestras de infantil), para asegurar el desarrollo de conocimientos didácticos y disciplinares necesarios para enseñar las ideas elementales de estadística propias del nivel de Educación Infantil. En este sentido, el Ministerio de Educación chileno (MINEDUC, 2012), a través del Centro de Perfeccionamiento, Experimentación e Investigaciones Pedagógicas (CPEIP), estableció en los Estándares orientadores para egresados de carreras de Educación Parvularia, los conocimientos pedagógicos y disciplinares que deben poseer todas las maestras que egresen de algún programa de formación en Chile. Estos estándares se clasifican en pedagógicos y disciplinares. En el primer caso, uno de los indicadores relacionados al estándar 3 (comprende el currículo de Educación Parvularia) es "conoce los objetivos y contenidos de los diferentes sectores de aprendizaje del primer nivel de enseñanza básica" (MINEDUC, 2012). En segundo lugar, el estándar disciplinar 6 (maneja estrategias pedagógicas basadas en su comprensión de las nociones fundamentales de las matemáticas), explicita que las maestras logren el objetivo de "comprender nociones de datos y azar: estadística descriptiva, conceptos básicos de probabilidades y sistemas de representación de información cuantificable" (MINEDUC, 2012). Ambos estándares, evidencian el conocimiento sobre estadística que debería tener una Maestra de infantil.

Desde la literatura, diversos investigadores han propuesto modelos para caracterizar el conocimiento matemático que se necesita para enseñar esta disciplina. En particular, Ball et al. (2008) proponen el Mathematical Knowledge for Teaching (MKT), donde se definen los distintos conocimientos que debe demostrar el profesor para apoyar el desarrollo de la competencia matemática de sus estudiantes. Este modelo considera, por ejemplo, el conocimiento especializado del contenido (SCK), que permite al maestro participar en tareas de enseñanza, incluyendo variedad de formas para representar las ideas, el entregar explicaciones matemáticas claras y precisas, comprender y aplicar diversos modelos y/o métodos en la resolución de problemas (Hill et al., 2008). En este contexto, diversas investigaciones han abordado aspectos del conocimiento matemático en profesores de Educación Primaria (en formación o en activo); sin embargo, son escasos los estudios que abordan esta temática en maestros de Educación Infantil.

Lee (2017) analizó el conocimiento pedagógico del contenido que evidenciaban treinta maestros de Educación Infantil en matemática, observando cómo advierten las situaciones matemáticas en que los niños se involucran y cómo interpretan la actividad matemática de los niños. Concluye que los maestros requieren mayores niveles de conocimiento para reconocer conceptos matemáticos específicos y poder potenciar el pensamiento matemático de los niños. De igual forma, Samuel et al. (2018) estudian el conocimiento matemático inicial de futuras maestras de Educación Infantil al analizar tareas escolares sobre simetría. En sus resultados, reconocen debilidades en cuanto al dominio del conocimiento matemático, aspecto que dificulta la interpretación de los errores en las producciones de los niños. Al respecto, Korkmaz y Şahin (2020), al examinar el conocimiento del contenido pedagógico en estudiantes para maestros de Educación Infantil sobre las formas geométricas en el contexto de los errores de los niños, observan que los futuros maestros no tendrían la capacidad para identificar los errores de los niños y para proponer soluciones que permitan reconstruir aprendizajes matemáticos. 
Tirosh et al. (2019) investigan el conocimiento del contenido matemático de maestros de Educación Infantil sobre patrones, la estructura y las explicaciones matemáticas proporcionadas por ellos, encontrando que estos son capaces de desarrollar tareas básicas en relación al tema, sin evidenciar una comprensión profunda del tema que permita dar explicaciones matemáticas respecto de la estructura del patrón y los distintos modos de representarlo. Para ello, Björklund y Barendregt (2016) señalan la necesidad de hacer explícito cómo el conocimiento pedagógico del contenido puede ser reconocido en una práctica y, a la vez, cómo puede ser implementado para construir conocimientos y contenidos matemáticos específicos. A parir de dos situaciones de enseñanza Pacheco-Figuereido et al. (2018), exploran la especificidad del conocimiento del contenido pedagógico y cómo este es movilizado en estudiantes para maestros. Los resultados sugieren que, aunque existe un fuerte componente de conocimiento del contenido, los futuros profesores todavía tienen un conocimiento limitado y desconectado de temas matemáticos

Así mismo, Leavy y Hourigan (2018) analizan una actividad elaborada por futuros maestros de infantil, que consistía en el diseño, implementación y rediseño de cinco lecciones sobre la iniciación a la construcción del número para niños de 4 a 5 años de edad. Los resultados evidencian lo complejo de la actividad, dado que los maestros no lograron demonstrar un conocimiento matemático especializado para mostrar representaciones de conceptos numéricos, ni utilizar un lenguaje matemático específico para responder a las tareas. La dificultad que tienen los maestros de infantil, en activo o en formación, para construir aprendizajes matemáticos es evidenciada en diferentes estudios, como los de Parks y Wager (2015) y Varol et al. (2012), en los que se señala que estos tienen un débil conocimiento de la matemática, así como de la forma en que se enseña en edades tempranas. Desde la perspectiva de Tirosh et al. (2020) resulta relevante ampliar la investigación del conocimiento matemático de diferentes conceptos en maestros de infantil.

Los estudios centrados en Educación Infantil son escasos, más aún aquellos relacionados con temas de estadística. Uno de estos trabajos es el realizado por Díaz-Levicoy et al. (2016), donde se analizan los niveles de lectura alcanzados por 121 futuras maestras de Educación Infantil. Los resultados muestran que el mayor porcentaje de éxito de las futuras maestras están asociadas a las preguntas que exigen una respuesta literal (93.4\%) y las que demandan operaciones aritméticas sencillas (45.5\%). Solo un $7.4 \%$ es capaz de realizar una producción basada en los datos del gráfico. Debido a la escasez de estudios centrados en los temas de estadística en Educación Infantil, es que nos planteamos el objetivo de evaluar el conocimiento especializado sobre gráficos estadísticos por futuras maestras de Educación Infantil.

\section{METODOLOGÍA}

Esta investigación sigue una metodología cualitativa (Cooley, 2013), de nivel descriptivo y se utiliza el análisis de contenido (Bengtsson, 2016) como método para estudiar las respuestas entregadas por las futuras maestras de Educación Infantil. La muestra considerada en esta investigación fue de tipo intencional y estuvo formada por 102 futuras maestras de Educación Infantil pertenecientes a dos universidades de la zona central de Chile, constituida en su totalidad por mujeres, quienes respondieron voluntariamente al cuestionario en un tiempo máximo de dos horas pedagógicas y previa firma de un consentimiento informado. El instrumento aplicado buscó evaluar el conocimiento matemático (estadístico) para la enseñanza de los gráficos estadísticos, cuyo proceso de diseño y validación se describe en Samuel et al. (2019).

En este caso, reportamos cuatro ítems relacionados con el conocimiento especializado del contenido (figura 1): en el primer ítem se pide definir gráfico estadístico; en el segundo se pide hacer un listado con los conocimientos matemáticos y estadísticos usados en el trabajo con un gráfico de barras; en el tercer ítem se pide responder a cinco preguntas relacionadas con la identificación de tipo de gráfico y lecturas con diferentes niveles de dificultad; y en el cuarto ítem se pide mencionar el tipo de gráfico que usaría para mostrar la información representada por un estudiante. Las respuestas entregadas por las futuras maestras se codificaron según la calidad de las mismas, generando diferentes categorías-correcta, parcialmente correcta e incorrecta; o destacado, competente, básico e insatisfactorio. Para mencionado análisis, se utiliza el código $\mathrm{FMI} X$, donde $\mathrm{FMI}$ significa futuras maestras de infantil y $X$ corresponde al número asignado al orden en que se revisó cada instrumento, es decir, FMI99 corresponde a la respuesta de la futura maestra que se analizó en el lugar 99.

\section{RESULTADOS}

A continuación, se presentan los resultados asociados a cada uno de los ítems relacionados con el conocimiento especializado del contenido sobre gráficos estadísticos por futuras maestras de Educación Infantil.

\section{Ítem 1. Definición de gráfico estadístico}

El primer ítem (¿Cómo definiría usted un gráfico estadístico?) está relacionado con el conocimiento especializado del contenido, porque la futura maestras debe mencionar elementos que lo caracterizan: 1) manifestar un conocimiento profundo y específico sobre gráficos estadísticos, es decir, define con precisión 
este objeto estadístico, mencionando su utilidad y la forma de analizarlo; 2) representar con exactitud las ideas sobre gráficos estadísticos, esto es, comprende la función de cada elemento de los diferentes tipos de representaciones gráficas; 3) ofrecer explicaciones precisas sobre el uso o aplicación de los gráficos estadísticos, a saber, se indica las situaciones en que aparecen y se aplican estas representaciones. Para analizar este ítem se han establecido los siguientes tres niveles de dominio de conocimiento (tabla 1).

Ítem 1. ¿Cómo definiría usted un gráfico estadístico?

Ítem 2. Haga una lista considerando los conocimientos matemáticos y estadísticos que usa cuando trabaja con un gráfico de barras.

Ítem 3. En el siguiente gráfico se muestra el nivel de aprobación y reprobación (en porcentaje: \%) del desempeño de la directora del jardín infantil "Caracolito" durante los últimos nueve meses.

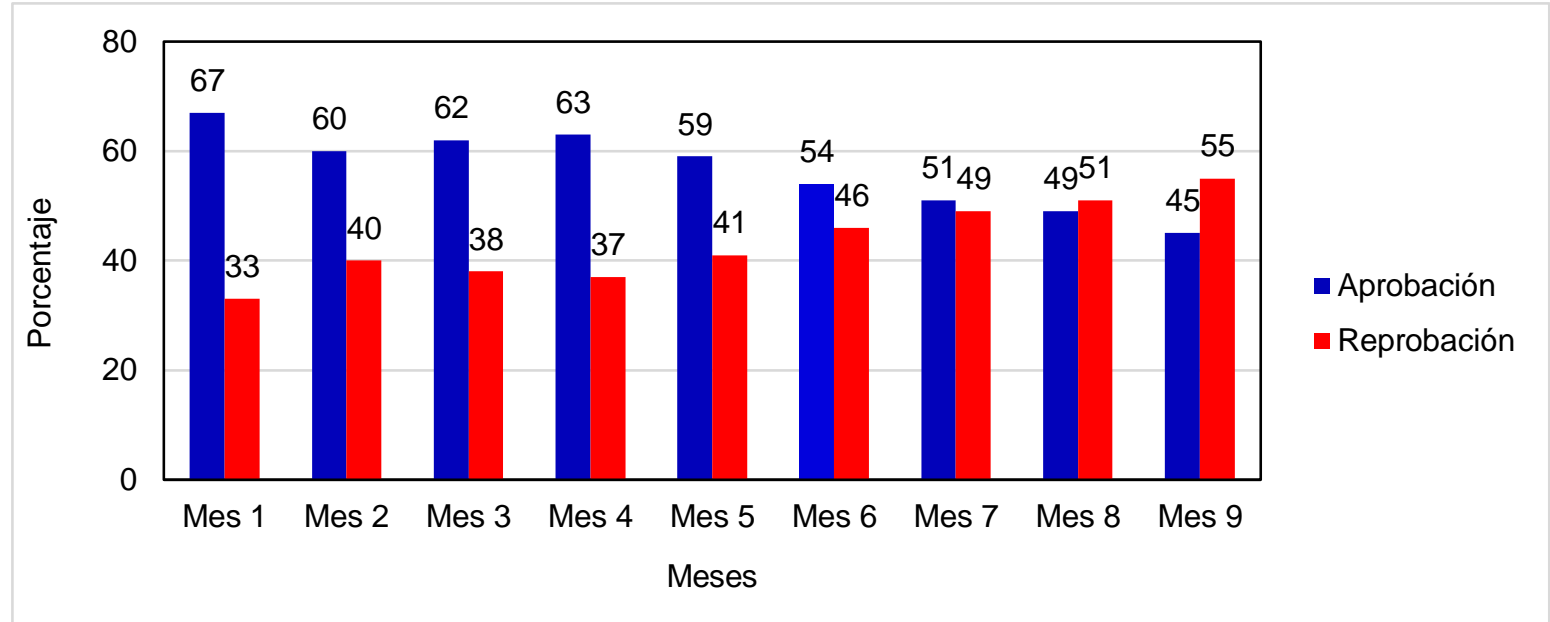

De acuerdo con la información mostrada en el gráfico, responda las siguientes preguntas.

a. Indique el tipo de gráfico.

b. ¿Qué porcentaje de reprobación alcanzó la directora del jardín infantil en el Mes 5 ?

c. ¿Se observa algún tipo de tendencia en relación al nivel de aprobación y reprobación de la directora del jardín infantil? Explique brevemente.

d. ¿Cuál es el promedio de aprobación de la gestión durante los nueve meses?

e. ¿Cuál será el porcentaje de aprobación que tendría la directora del Jardín en el décimo mes? Explique cómo lo obtuvo.

Ítem 4. Una educadora dio a los niños de kínder la tarea de preguntar a los miembros de su familia sobre sus insectos favoritos. Las respuestas de uno de los niños se muestran en el siguiente diagrama.

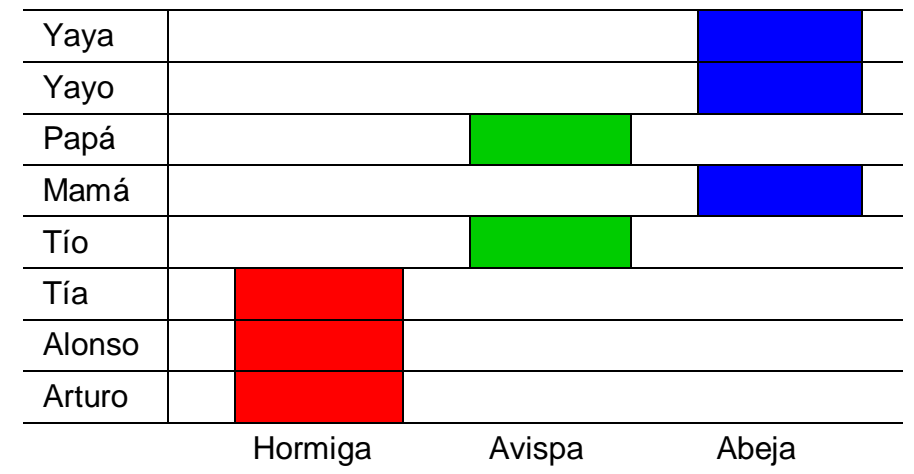

¿Qué gráfico estadístico usarías para representar esta información?

Fig. 1: Ítems utilizados en el estudio 
Tabla 1: categorías, descripción, ejemplos y frecuencia (y porcentaje) asociados al ítem 1

\begin{tabular}{|c|c|c|c|}
\hline $\begin{array}{l}\text { Nivel de } \\
\text { dominio }\end{array}$ & Descripción & Ejemplo & $\begin{array}{c}\text { Frecuencia } \\
\text { (y porcentaje) }\end{array}$ \\
\hline Destacado & $\begin{array}{l}\text { Considera los tres indicadores } \\
\text { para describir el conocimiento } \\
\text { especializado sobre gráficos } \\
\text { estadísticos }\end{array}$ & $\begin{array}{l}\text { Definiría un gráfico estadístico como un } \\
\text { instrumento que permite representar datos en } \\
\text { relación a un tema de estudio y que } \\
\text { demuestra la frecuencia o cantidad de } \\
\text { elementos respecto a un tema investigado } \\
\text { (FMI30). } \\
\text { Un gráfico estadístico es el que representa } \\
\text { los resultados obtenidos a partir de hechos o } \\
\text { sucesos concretos y que necesitan un } \\
\text { análisis de la información, facilitando su } \\
\text { interpretación (FMI41). }\end{array}$ & $5(4,90)$ \\
\hline Competente & $\begin{array}{l}\text { Considera dos indicadores del } \\
\text { conocimiento especializado } \\
\text { sobre gráficos estadísticos, } \\
\text { incluso con imprecisiones }\end{array}$ & $\begin{array}{l}\text { Es un instrumento que nos permite observar } \\
\text { y analizar diversos resultados de algo } \\
\text { (FMI61). } \\
\text { Instrumento que se utiliza para ordenar y } \\
\text { representar datos respecto a el objeto en } \\
\text { estudio (FMI44). }\end{array}$ & $11(10,78)$ \\
\hline Básico & $\begin{array}{l}\text { Considera solo un indicador del } \\
\text { conocimiento especializado } \\
\text { sobre gráficos estadísticos, } \\
\text { incluso con imprecisiones }\end{array}$ & $\begin{array}{l}\text { Son datos representados a través de un } \\
\text { gráfico (FMI90). } \\
\text { Como un instrumento para medir una } \\
\text { población, una frecuencia de algo, dinero, } \\
\text { etc. Que muestra que es lo que más abunda } \\
\text { y lo que no (FMI95) }\end{array}$ & $39(38,24)$ \\
\hline Insatisfactorio & $\begin{array}{l}\text { Considera elementos confusos, } \\
\text { alejados de lo mencionado en } \\
\text { los indicadores, y con errores } \\
\text { importantes }\end{array}$ & $\begin{array}{l}\text { Un dibujo mediante el cual se muestran } \\
\text { patrones del objeto a estudiar más fácil de } \\
\text { entender (FMI97). } \\
\text { Un gráfico estadístico son rectas que nos } \\
\text { ayudan a resumir más fácil algunos datos } \\
\text { entregados (FMl102). }\end{array}$ & $41(40,20)$ \\
\hline No responde & \multicolumn{2}{|c|}{ La actividad no es abordada por la futura maestra de infantil } & $6(5,88)$ \\
\hline & & Total & $102(100)$ \\
\hline
\end{tabular}

\section{Ítem 2. Lista de conocimientos matemáticos y estadísticos}

En el segundo ítem se espera que las futuras maestras hagan un listado en el que se identifiquen los elementos matemáticos y estadísticos que intervienen al trabajar con un gráfico de barras. En este sentido, en primer lugar, se cuantifica la cantidad de palabras que ellas mencionan, sin discriminar entre buenas y malas. La tabla 2 muestra que un $22.55 \%$ de las futuras maestras no responde a la actividad formulada, el $21.57 \%$ mencionan tres conceptos y el $15.69 \%$ expresan cuatro.

Tabla 2: Frecuencia y porcentaje de la cantidad de palabras o expresiones listado por las futuras maestras de infantil

\begin{tabular}{|l|c|c|}
\hline $\begin{array}{l}\text { Cantidad de } \\
\text { términos }\end{array}$ & Frecuencia & Porcentaje \\
\hline 1 & 2 & 1,96 \\
\hline 2 & $11-1$ & 10,78 \\
\hline 3 & 22 & 21,57 \\
\hline 4 & $16+1$ & 15,69 \\
\hline 5 & 10 & 9,80 \\
\hline 6 & 4 & 3,92 \\
\hline 7 & 2 & 1,96 \\
\hline 8 & 1 & 0,98 \\
\hline 10 & 1 & 0,98 \\
\hline Frases & 10 & 9,80 \\
\hline No responde & 23 & 22,55 \\
\hline Total & 102 & 100 \\
\hline
\end{tabular}


De modo complementario, se contabilizan el listado de conceptos que se consideran correctos, los que se resumen en la tabla 3 . En ella, se pueden identificar 14 conceptos relacionados con el contenido matemático y 6 en el estadístico. En el primer caso, los conceptos más frecuentes son cantidad, mencionado por 27 (27.54\%) futuras maestras; así como números, mencionado por 16(16.32\%). En el segundo caso, los términos más nombrados son datos (20.4\%), seguido de rango y moda $(8.16 \%)$

Tabla 3: Frecuencia y porcentaje de conceptos matemáticos y estadísticos listados por las futuras maestras

\begin{tabular}{|c|c|c|}
\hline \multicolumn{3}{|c|}{ Conocimiento matemático } \\
\hline Concepto & Frecuencia & Porcentaje \\
\hline Contar & 6 & 6,12 \\
\hline Clasificar & 6 & 6,12 \\
\hline Ordenar & 3 & 3,06 \\
\hline Recta (línea) & 1 & 1,02 \\
\hline Proporcionalidad & 2 & 2,04 \\
\hline Plano cartesiano & 3 & 3,06 \\
\hline Cantidad & 27 & 27,54 \\
\hline Correspondencia & 2 & 2,04 \\
\hline Escala & 2 & 2,04 \\
\hline Mínimo & 1 & 1,02 \\
\hline Máximo & 1 & 1,02 \\
\hline Menor, mayor o igual & 2 & 2,04 \\
\hline Menos que o más que & 1 & 1,02 \\
\hline Número & 16 & 16,32 \\
\hline \multicolumn{3}{|c|}{ Conocimiento estadístico } \\
\hline Concepto & Frecuencia & Porcentaje \\
\hline Dato & 20 & 20,4 \\
\hline Frecuencia & 6 & 6,12 \\
\hline Variable & 6 & 6,12 \\
\hline Rango & 8 & 8,16 \\
\hline Moda & 8 & 8,16 \\
\hline Tendencia de los datos & 1 & 1,02 \\
\hline Información & 3 & 3,06 \\
\hline
\end{tabular}

\section{Ítem 3. Lectura de un gráfico de barras dobles}

El tercer ítem consta de cinco preguntas, referidas a la identificación del tipo de representación, lectura literal de la información, identificación de tendencia de los datos, cálculo de promedio y, por último, realización de predicciones, las que avanzan en su nivel de complejidad. En la primera pregunta, las futuras maestras de infantil deben identificar el tipo de representación en que se resumen los datos. Como se muestra en la tabla 4, las respuestas de las futuras maestras, en su mayoría, son consideradas correctas $(92.16 \%)$, entre las respuestas se encuentran: "Gráfico de barras" (FMI13); "gráfico de barra" (FMI23); "de barras" (FMI93). En segundo lugar, se encuentran aquellas respuestas consideradas como incorrectas (4.90\%), como, por ejemplo: "gráfico porcentual" (FMI63), "comparativo" (FMI75). Finalmente, solo la respuesta de FMI77 se ha clasificado como parcialmente correcta $(0.98 \%)$, por la imprecisión en la identificación del objeto matemático "Gráfico estadístico y de barras".

Tabla 4: Frecuencia y porcentaje del éxito en la pregunta 3a (tipo de gráfico)

\begin{tabular}{|l|c|c|}
\hline Tipo de respuesta & Frecuencia & Porcentaje \\
\hline Correcta & 94 & 92.16 \\
\hline Parcialmente correcta & 1 & 0.98 \\
\hline Incorrecta & 5 & 4.90 \\
\hline No responde & 2 & 1.96 \\
\hline Total & 102 & 100 \\
\hline
\end{tabular}


Las distribuciones de las respuestas para la segunda pregunta se resumen en la tabla 5. En ella, observamos que las correctas son las que predominan, con un $88.24 \%$ de las respuestas, identificando entre ellas: "un $41 \%$ de reprobación" (FMI90); "alcanzó un 41\%" (FMI20); y "41\%" (FMI71). En segundo lugar, están aquellas respuestas que se han clasificado como incorrectas (5.88\%), es decir, en las que los valores no concuerdan con los observados en el gráfico, por ejemplo, "40\%" (FMI16); "59\% de porcentaje" (FMI62); y "59\%" (FMI80). Finalmente, el $4.90 \%$ de las respuestas de las futuras maestras se han considerado como parcialmente correctas, es decir, presentan imprecisiones en sus respuestas, ya sea la ausencia de símbolos porcentuales o su forma de notarlos: "41" (FMI69); "41 de reprobación" (FMI90); "\%41" (FMI92).

Tabla 5: Frecuencia y porcentaje del éxito en la pregunta $3 \mathrm{~b}$ (lectura reprobación en el mes 5)

\begin{tabular}{|l|c|c|}
\hline Tipo de respuesta & Frecuencia & Porcentaje \\
\hline Correcta & 90 & 88.24 \\
\hline Parcialmente correcta & 5 & 4.90 \\
\hline Incorrecta & 6 & 5.88 \\
\hline No responde & 1 & 0.98 \\
\hline Total & 102 & 100 \\
\hline
\end{tabular}

En la tabla 6 se muestra la distribución de la tercera pregunta relacionada con la identificación, si existe, de alguna tendencia entre los porcentajes de aprobación y reprobación por la gestión de la directora del jardín infantil. En tipo de respuesta que predomina es la de incorrectas (33.33\%), donde las futuras maestras no mencionan la tendencia de quienes aprueban la gestión (disminuye) o de su reprobación (aumenta), por ejemplo: "No se observa tendencias" (FMI17); "Sí, en el mes 7 y 8 ya que ambos poseen igual número de aprobación y reprobación" (FMI16); "El mes 7 y 8 repiten sus cifras, pero cambiar el orden" (FMI98); "Sí, la tendencia es de aproximadamente 20" (FMI69).

Tabla 6: frecuencia y porcentaje del éxito en la pregunta 3c (tendencia en la aprobación y la reprobación)

\begin{tabular}{|l|c|c|}
\hline Tipo de respuesta & Frecuencia & Porcentaje \\
\hline Correcta & 26 & 25,49 \\
\hline Parcialmente correcta & 26 & 25,49 \\
\hline Incorrecta & 34 & 33,33 \\
\hline No responde & 16 & 15,69 \\
\hline Total & 102 & 100 \\
\hline
\end{tabular}

En segundo lugar, con un $25.49 \%$ están las respuestas correctas y parcialmente correctas, algunas clasificadas en el primer caso son: "Tiende a disminuir el nivel de aprobación y a aumentar el nivel de reprobación" (FMI10); "Ocurre que el nivel de aprobación comienza a bajar el porcentaje y el nivel de reprobación comienza a subir" (FMl13); "Desde el mes 4 hacia adelante, se puede observar una tendencia de reprobación en aumento, así mismo, ocurre con la aprobación que va disminuyendo" (FMI23); "En relación al nivel de aprobación esta tiende a disminuir a lo largo de los meses y el nivel de reprobación tiende a aumentar" (FMI26). Algunos ejemplos de respuestas parcialmente correctas son: "Tiene como tendencia la disminución de la aprobación" (FMI40); "Cuando el nivel de reprobación va aumentando" (FMI82); "En el transcurso de los meses la reprobación va en aumento" (FMl101). Se puede observar que estas respuestas solamente hacen referencia a la aprobación o a la reprobación, incluso con algunas imprecisiones.

La tabla 7 muestra la distribución de las respuestas de las futuras maestras de Educación Infantil, donde se observa que las respuestas con mayor predominio son las clasificadas como incorrectas (40,04\%), es decir, el valor entregado no es el correcto y no especifica el proceso para obtenerlo. Ejemplo de estas respuestas son: "el promedio es de 53\%" (FMI20); "El promedio de aprobación de la gestión es alrededor de un 55 a un 65\%" (FMI48); "El promedio de aprobación durante los nueves meses sería de 5,7" (FMI65); "El promedio de aprobación es un 59\% más o menos" (FMI68). En segundo lugar, están las respuestas correctas (22.55\%), donde se entrega el valor correspondiente a la media aritmética de los porcentajes de aprobación, aunque no hagan explicito el desarrollo de la actividad. Ejemplo de estas respuestas son: "El promedio de aprobación es de 56.6\%" (FMI14); "56.6\%" (FMI44); "El promedio de aprobación de la gestión durante los 9 meses es de 56.6\%" (FMI52). El 10.78\% de las respuestas se consideran como parcialmente correctas, entre ellas se consideran principalmente las con problemas de notación, es decir, no se especifica que están trabajando con porcentajes, por ejemplo: "El promedio de aprobación es de 56.6 (510:9=56.6)" (FMI7); "56,6" (FMI33); "El promedio de los nueves meses es de un 56,6" (FMI60). 
Tabla 7: frecuencia y porcentaje del éxito en la pregunta 3d (promedio de aprobación)

\begin{tabular}{|l|c|c|}
\hline Tipo de respuesta & Frecuencia & Porcentaje \\
\hline Correcta & 23 & 22,55 \\
\hline Parcialmente correcta & 11 & 10,78 \\
\hline Incorrecta & 49 & 40,04 \\
\hline No responde & 19 & 18,63 \\
\hline Total & 102 & 100 \\
\hline
\end{tabular}

En la tabla 8 se resume los resultados de la quinta pregunta, la que tiene relación con la predicción de un valor que no está representado en el gráfico estadístico, es decir, estimar el porcentaje de aprobación durante el décimo mes. De ella se observa que el $81.37 \%$ de las futuras maestras no responde a la pregunta formulada, además de no encontrar respuestas consideradas como correctas. El 14.71\% de las respuestas se consideran como incorrectas, es decir, los valores entregados no presentan sentido en el contexto de los datos o son valores que se alejan del esperado, y no explican el procedimiento seguido. Algunos ejemplos de estas respuestas son: " $57 \%$ promediando todos los porcentajes de aprobación de los meses anteriores" (FMI17); "Tendríamos que saber cuántas personas" (FMI36); "Aumentaría, pero no se calcular cuánto" (FMI76); "No se puede saber, ya que los resultados de un mes son totalmente independientes de otro" (FMI85). Finalmente, solo $3.92 \%$ de las futuras maestras entregan respuestas consideradas como parcialmente correctas, es decir, entregan valores siguiendo la tendencia de los datos, pero hay imprecisión al indicar el proceso seguido, por ejemplo: "El porcentaje de aprobación fluctuará entre $43 \%$ y $41 \%$, lo obtuve sacando la tendencia que existía entre los últimos 4 meses" (FMI3); "43\% intuición siguiendo el patrón de descenso" (FMI53).

Tabla 8: frecuencia y porcentaje del éxito en la pregunta 3e (aprobación en el mes diez)

\begin{tabular}{|l|c|c|}
\hline Tipo de respuesta & Frecuencia & Porcentaje \\
\hline Correcta & 0 & 0 \\
\hline Parcialmente correcta & 4 & 3,92 \\
\hline Incorrecta & 15 & 14,71 \\
\hline No responde & 83 & 81,37 \\
\hline Total & 102 & 100 \\
\hline
\end{tabular}

\section{Ítem 4. Identificar un gráfico estadístico para representar datos}

En el último ítem, se pide nombrar un gráfico estadístico pertinente para representar variables cualitativas, según la información mostrada en el diagrama construido por un niño y donde las futuras maestras deben recurrir a su conocimiento especializado para responder a lo requerido. Las respuestas de las futuras maestras se han categorizado (tabla 8), mayoritariamente, como correctas (66.67\%), mientras que alrededor de un $20 \%$ no aborda la actividad. Entre las respuestas consideradas como correctas tenemos: "utilizaría un gráfico de barras o pictograma" (FMI34); "un gráfico circular" (FMI52); "gráfico de barras" (FMI90). Entre las respuestas parcialmente correctas están consideradas aquellas que mencionan un gráfico correcto y otro incorrecto o imprecisiones al identificarlos, por ejemplo, "un gráfico en círculos no conozco el nombre" (FMl16), "un gráfico de barras o puntos" (FMI60); "gráfico de barras o grafico lineal" (FMI92). Finalmente, se observan respuestas incorrectas, donde se nombra un gráfico errado o inexistente, entre ellas: "gráfico de tabla" (FMI18); "yo usaría una tabla o en su defecto un gráfico lineal" (FMI51); "yo utilizaría un gráfico de datos" (FMI56); "un gráfico de líneas" (FMI66).

Tabla 9: Frecuencia y porcentaje de tipo de respuestas al ítem 4

\begin{tabular}{|l|c|c|}
\hline Tipo de respuesta & Frecuencia & Porcentaje \\
\hline Correcta & 68 & 66.67 \\
\hline Parcialmente correcta & 5 & 4.90 \\
\hline Incorrecta & 10 & 9.80 \\
\hline No responde & 19 & 18.63 \\
\hline Total & 102 & 100 \\
\hline
\end{tabular}

\section{DISCUSIÓN}

La introducción de la estadística ha generado desafíos importantes, entre ellos, su enseñanza desde los primeros años (Educación Infantil y Primaria). Esta situación demanda maestros y profesores con el conocimiento didáctico y disciplinar, para desarrollar en sus estudiantes aspectos de cultura estadística que 
serán de utilidad para su convivencia en sociedad. En este sentido, los resultados de esta investigación, permiten una primera aproximación al conocimiento de futuras maestras de Educación Infantil sobre gráficos estadísticos en el contexto chileno, donde se aportan resultados novedosos y que pueden impactar en la formación que reciben en la universidad.

Respecto de los resultados, en el ítem 1 se observa que la mayoría de las futuras maestras de Educación Infantil entregan una definición de gráfico estadístico que son consideradas como insatisfactorias (38.24\%) y básicas $(40.20 \%)$, las que concentran en torno al $80 \%$ de ellas. En estas respuestas se reconocen ideas confusas, erradas o alejadas del conocimiento especializado sobre gráficos estadísticos, o indican solo un elemento. Estos resultados llaman la atención por tratarse de un concepto elemental, parte esencial de las nociones básicas de los sistemas de representación definidos en los estándares orientadores (MINEDUC, 2012).

En el ítem 2, las futuras maestras de infantil entregan un listado de conocimientos matemáticos más variados que los estadísticos. En el primer caso, destacan las ideas de cantidad (27.54\%) y número (16.32\%), mientras que el segundo caso está el concepto de dato (20.4\%). En general, los listados de conocimientos están relacionados al ámbito numérico, el que tiene mayor presencia en los estándares formadores (MINEDUC, 2012) y en el currículo de Educación Infantil (MINEDUC, 2018).

En el ítem 3 se presentan cinco tareas, en la primera el 92.16\% indica correctamente el tipo de gráfico, al indicar que se trata de un gráfico de barras, pero no especifican que es de barras dobles. En la segunda tarea, el $88.24 \%$ lee correctamente el porcentaje de reprobación en el mes 5. Luego, en la tercera tarea, la mayoría de las futuras maestras respondieron de manera incorrecta (33.33\%), es decir, no logran identificar la tendencia de los datos. De igual manera, en la cuarta tarea, se observa que las respuestas de un $40 \%$ de las futuras maestras son incorrectas, es decir, no calculan correctamente el promedio de los datos. Finalmente, en la tarea cinco, que pedía realizar una predicción de acuerdo con los datos mostrados en el gráfico, el $14.71 \%$ responde de manera incorrecta y el $81.37 \%$ no aborda la tarea. Estos resultados coinciden con lo reportado en Díaz-Levicoy et al. (2016).

Finalmente, en el ítem 4, el $66.67 \%$ de las futuras maestras identifica correctamente uno de los gráficos que se puede utilizar para representar la información mostrada, limitándose en su mayoría a mencionar el gráfico de barras, cuya estructura se visualiza en la representación del niño. Además, este tipo de gráfico tiene amplia presencia en los medios de comunicación (Cavalcanti et al., 2010), así como en el currículo y los libros de texto (Arteaga et al., 2020).

Estos resultados advierten que el conocimiento especializado sobre gráficos estadísticos de las futuras maestras de Educación Infantil que han participado en esta investigación, es insuficiente para responder a las situaciones planteadas, coincidiendo con estudios que abordan otros temas matemáticos (Korkmaz y Şahin, 2020; Pacheco-Figuereido et al., 2018; Samuel et al., 2018). Esta situación demanda revisar los planes formativos de las futuras maestras de Educación Infantil, de modo de asegurar un conocimiento más sólido de la matemática en general y de la estadística en particular.

\section{CONCLUSIONES}

Las conclusiones derivadas de los resultados son:

1) Las futuras maestras de Educación Infantil no evidencian una clara concepción referida a gráfico estadístico.

2) Los conocimientos declarados por las futuras maestras participes de la investigación están asociados mayoritariamente al ámbito numérico.

3) Las futuras maestras abordan con mayor facilidad aquellas preguntas relacionadas con la identificación de un tipo de gráfico y lectura literal.

\section{REFERENCIAS}

Alsina, Á., Contextos y propuestas para la enseñanza de la estadística y la probabilidad en educación infantil, Épsilon, 95, 25-48 (2017)

Arteaga, P., Díaz-Levicoy, D., y Batanero, C., Assessing Chilean primary school children's understanding of statistical graphs, https://doi.org/10.17648/acta.scientiae.5884, Acta Scientiae, 22(2), 2-24 (2020)

Ball, D., Thames, M., y Phelps, G., Content knowledge for teaching: what makes it special?,

https://doi.org/10.1177/0022487108324554, Journal of Teacher Education, 59(5), 389-407 (2008) 
Bengtsson, M., How to plan and perform a qualitative study using content analysis, https://doi.org/10.1016/j.npls.2016.01.001, NursingPlus Open, 2, 8-14 (2016)

Björklund, C., y Barendreg, W., Teachers' pedagogical mathematical awareness in swedish early childhood education, https://doi.org/10.1080/00313831.2015.1066426, Scandinavian Journal of Educational Research, 60(3), 359-377 (2016)

Cavalcanti, M. R., Natrielli, K. R., y Guimarães, G., Gráficos na mídia impressa, Boletim de Educação Matemática, 23(36), 733-751 (2010)

Cooley, A., Qualitative research in education: the origins, debates, and politics of creating knowledge, https://doi.org/10.1080/00131946.2013.783834, Educational Studies, 49(3), 247-262 (2013)

Del Pino, G., y Estrella, S., Educación estadística: Relaciones con la matemática, Pensamiento Educativo. Revista de Investigación Educacional Latinoamericana, 49(1), 53-64 (2012)

Díaz-Levicoy, D., Batanero, C., y otros dos autores, Chilean children's reading levels of statistical graphs, https://doi.org/10.29333/iejme/5786, International Electronic Journal of Mathematics Education, 14(3), 689-700 (2019)

Díaz-Levicoy, D., Sepúlveda, A., y otros dos autores, Lectura de tablas estadísticas por futuras maestras de Educación Infantil, Educação Matemática Pesquisa, 18(3), 1099-1115 (2016)

Gal, I., Does CensusAtSchool develop statistical literacy?, https://doi.org/10.3233/SJI-2011-0737, Statistical Journal of the IAOS, 27(3-4), 229-230 (2011)

Gould, R., Data literacy is statistical literacy, Statistics Education Research Journal, 16(1), $22-25$ (2017)

Hill, H. C., Ball, D. L., y Schlling, S. G., Unpacking pedagogical content knowledge of students, Journal for Research in Mathematics Education, 39(4), 372-400 (2008)

Jurečková, M., y Csachová, L., Statistical literacy of Slovak lower secondary school students, Technium Social Sciences Journal, 9, 163-173 (2020).

Korkmaz, H. I., y Şahin, Ö., Preservice preschool teachers' pedagogical content knowledge on geometric shapes in terms of children's mistakes, https://doi.org/10.1080/02568543.2019.1701150, Journal of Research in Childhood Education, 34(3), 1-21(2020)

Leavy A., y Hourigan M., Using lesson study to support the teaching of early number concepts: examining the development of prospective teachers' specialized content knowledge, https://doi.org/10.1007/s10643-016-0834-6, Early Childhood Education Journal, 46(1), 47-60 (2018)

Lee, J. E., Preschool teachers' pedagogical content knowledge in mathematics, https://doi.org/10.1007/s13158-0170189-1, International Journal of Early Childhood, 49(2), 229-243 (2017)

Lipič, N., y Ovsenik, M., The effect of statistical literacy on response to environmental change, https://doi.org/10.2478/orga-2020-0010, Organizacija, 53(2), 147-163 (2020)

McConway, K., Statistics and the media: a statistician's view, https://doi.org/10.1177/1464884915593243, Journalism, 17(1), 49-65 (2016).

MINEDUC, Estándares orientadores para carreras de educación parvularia, Centro de Investigación Avanzada en Educación, Santiago, Chile (2012)

MINEDUC, Bases curriculares educación parvularia, Subsecretaría de Educación Parvularia, Santiago, Chile (2018)

Molina-Portillo, E., Contreras, J. M., y otros dos autores, Statistical literacy in the information society, Boletín de Estadística e Investigación Operativa, 35(2), 148-169 (2019)

Pacheco-Figueiredo, M., Gomes, H., y Rodrigues, C., Mathematical pedagogical content knowledge in early childhood education: tales from the 'great unknown' in teacher education in Portugal,

https://doi.org/10.1080/1350293X.2018.1487164, European Early Childhood Education Research Journal, 26(4), 535$546(2018)$

Parks, A., y Wager, A., What knowledge is shaping teacher preparation in early childhood mathematics?, https://doi.org/10.1080/10901027.2015.1030520, Journal of Early Childhood Teacher Education, 36(2), 124-141 (2015)

Samuel, M., Díaz-Levicoy, D., y Rodríguez-Alveal, F., Diseño y validación de un cuestionario para evaluar la comprensión de gráficos estadísticos en futuras educadoras de párvulos, Revista Espacios, 40(41), 20 (2019)

Samuel, M., Vanegas, Y., y Giménez, J., Caracterización del conocimiento matemático de futuras maestras de educación infantil, https://doi.org/10.13042/Bordon.2018.62907, Bordón, 70(3), 61-75 (2018)

Tirosh, D., Tsamir, P., y otros tres autores, Preschool teachers' knowledge of repeating patterns: focusing on structure and the unit of repeat, https://doi.org/10.1007/s10857-017-9395-x, Journal of Mathematics Teacher Education, 22(3), 305-325 (2019)

Varol, F., Farran, D., y otros tres autores, Professional development for preschool teachers: evidence for practice, https://doi.org/10.1080/15240754.2011.638742, NHSA Dialog, 15(1), 122-126 (2012)

Weiland, T., Problematizing statistical literacy: an intersection of critical and statistical literacies, Educational Studies in Mathematics, 96(1), 33-47 (2017) 